\title{
Ärztlich assistierter Suizid: \\ Kann „unerträgliches Leiden“ ein Kriterium sein?
}

\author{
Claudia Bozzaro
}

\begin{abstract}
Welche Umstände könnten den ärztlich assistierten Suizid legitimieren? Diese Frage steht aktuell ganz oben auf der politischen Agenda. Dabei taucht ein Begriff in dieser Debatte immer wieder auf: Der Begriff des „unerträglichen Leidens“. Darauf beziehen sich die Beteiligten mit großer Selbstverständlichkeit. Doch was genau bedeutet dieser Begriff? Und welche Folgen für die medizinische Praxis hat die fehlende begriffliche Schärfe?
\end{abstract}

\section{Hintergrund}

Ein klassisches Ziel | Hinweise auf „unerträgliches Leiden“ sind offenbar so überzeugend, dass Maßnahmen zur Leidenslinderung kaum weiter legitimiert werden müssen. Mit dem Wort „Leiden“ werden gewöhnlich negative Erfahrungen bezeichnet, die nicht sein sollten und die den unmittelbaren Impuls wecken, etwas gegen ihr weiteres Bestehen zu unternehmen. Dementsprechend definiert die Medizin das Lindern von Leiden als eines ihrer klassischen Ziele [5], was auch für die Begleitung Sterbender gilt [3].

Nötige Begriffsklärung | Das Ziel der Leidenslinderung ist grundsätzlich plausibel - erst recht, wenn Menschen aufgrund „unerträglichen Leidens“ an Suizid denken. Was aber genau soll dieser Begriff sagen? Diese Klärung ist wichtig, denn der Begriff ist mehr als nur ein rhetorisches Argument. Er hat sich zum festen Kriterium etabliert, das in verschiedenen medizinischen Kontexten praktisch angewandt wird.

- „Unerträgliches Leiden“ ist ein fester Bestandteil im niederländischen Gesetz zur Regelung der Lebensbeendigung auf Verlangen und der ärztlichen Beihilfe zum Suizid.

- Der Begriff ist auch für die Indikation der palliativen Sedierung von zentraler Bedeutung.

In beiden Kontexten gibt es Probleme und offene Fragen, wenn man den Leidensbegriff in der medizinischen Praxis anwendet [27, 31]. Das wäre hierzulande - bei Zulassung des ärztlich assistierten Suizids - wohl ähnlich. Eine begriffliche Auseinandersetzung ist dringend nötig.

\section{„Unerträgliches Leiden“ als Kriterium in der medizinischen Praxis}

Niederländische Gesetzgebung | Im Jahr 2002 verabschiedeten die Niederlande ihre Gesetzgebung zur Regelung der Lebensbeendigung auf Verlangen und der Hilfe bei der Selbsttötung. Da- nach ist für den Arzt Straffreiheit vorgesehen, wenn er folgende Sorgfaltskriterien einhält:

1. Er muss überzeugt sein, dass der Patient seine Bitte freiwillig und nach reiflicher Überlegung äußert.

2. Der Zustand des Patienten muss aussichtslos und sein Leiden unerträglich sein.

3. Der Patient muss über seine Situation und Aussichten aufgeklärt sein.

4. Beide müssen überzeugt sein, dass es für die Situation keine andere annehmbare Lösung gibt.

5. Ein zweiter Arzt muss die Sorgfaltskriterien überprüfen.

6. Der Arzt muss mit medizinischer Sorgfalt vorgehen [14].

Der Leidensbegriff hat eine hohe Relevanz in der niederländischen Gesetzgebung und steht gleich an zweiter Stelle - aber er wird nirgends definiert. Auch in der internationalen wissenschaftlichen Literatur zur niederländischen Praxis am Lebensende hat sich bis dato keine allgemein akzeptierte Definition durchgesetzt [32]. Es ist absolut offen, wie dieser Begriff interpretiert werden sollte.

Palliative Sedierung I In der Palliativmedizin hat sich der Einsatz palliativer Sedierungsmaßnahmen etabliert, u.a. auch die der tiefen und kontinuierlichen Sedierung (,terminale Sedierung“) [16]. Dabei dämpfen Medikamente komplett das Bewusstsein bis zum Todeseintritt.

- Diese Sedierung gilt als Maßnahme der ultima ratio. Sie soll lediglich in Extremfällen ohne weitere Möglichkeiten der Symptomkontrolle angewendet werden [8].

- Das Ziel dieser Sedierung ist es, „unerträgliches Leiden bei sonst therapierefraktären Symptomen zu lindern“ [24: 26; vgl. auch 21].

Auch hier hat der Begriff des „unerträglichen Leidens“ eine zentrale Rolle - nämlich bei der Indikationsstellung für diese Sedierung. Wieder fehlen belastbare Definitionen [17]. Diese mangelnde Schärfe des Leidensbegriffs führt bei seiner Anwendung in der medizinischen Praxis offenkundig zu Schwierigkeiten [33]. 


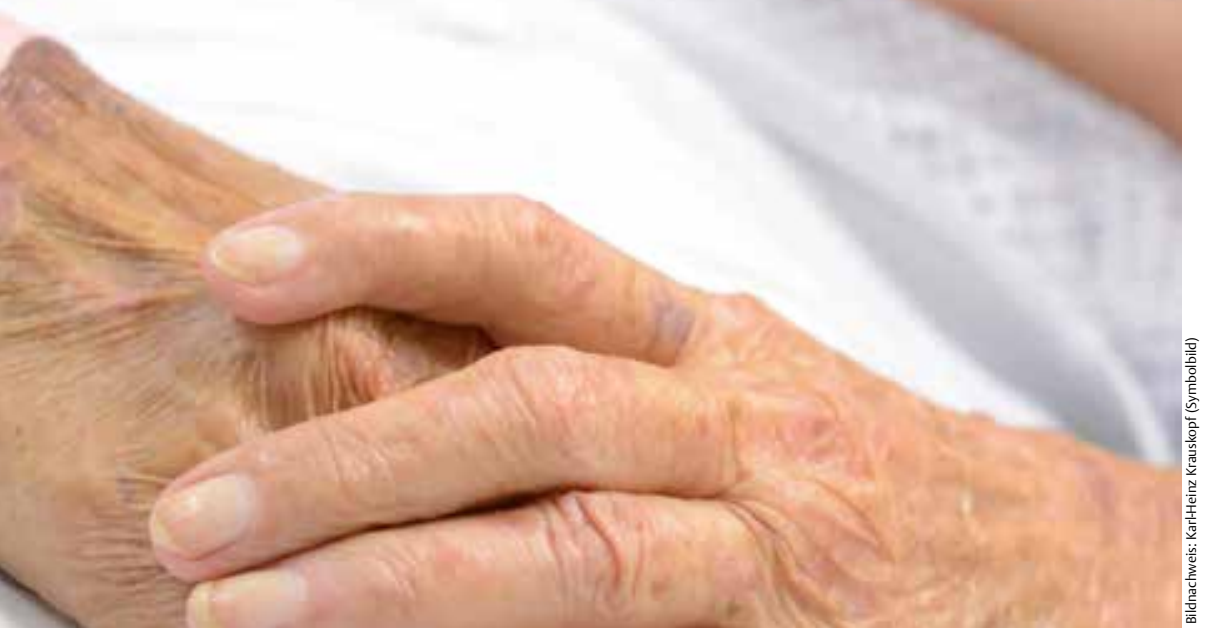

\section{Schwierigkeiten mit dem Leidens- begriff in der medizinischen Praxis}

Subjektivität des Leidens | Im niederländischen Kontext findet man nur sehr allgemeine Definitionen des Leidens, z.B. als „a subjective experience differing from pain “ $[1$, S. 825]. In einer der wenigen Definitionen aus dem palliativmedizinischen Kontext wird unerträgliches Leiden beschrieben als „die individuelle und subjektiv empfundene Intensität von Symptomen oder Situationen, deren andauerndes Empfinden bzw. Erleben so belastend ist, dass sie von einem Patienten nicht akzeptiert werden kann“ [22, S. 2734]. Beide Definitionen decken sich mit gängigen theoretischen Konzeptionen des Leidens als einem emotional sehr unangenehmen [10] und vor allem subjektiven Zustand [6].

- Die Definitionen betonen vor allem den subjektiven Charakter: Jedes Leiden hängt primär von der individuellen Empfindsamkeit und von subjektiven Bewertungsmaßstäben ab.

Die Deutungshoheit über das Leiden liegt primär beim Betroffenen - nur er weiß, unter welchen Symptomen und Situationen er in welchem Ausmaß leidet. Eine objektive Beurteilung des Leidens durch Ärzte, Kontrollkommissionen, Angehörige usw. ist faktisch unmöglich.

Ärzte brauchen zur objektiven Beurteilung eines „unerträglichen Leidens“ die subjektive Äußerung des Patienten. Nur die Äußerungen, nicht aber der Leidenszustand selbst, sind intersubjektiv im Gespräch zugänglich. Es ist offensichtlich aber ein Unterschied, ob der Patient das subjektiv empfundene Leiden äußern und vor allem dem Arzt plausibel machen kann oder ob ein solcher Zustand für den Arzt unmittelbar zugänglich und objektiv nachweisbar vorliegt. Dies führt zu Problemen, denn ein Arzt muss das „unerträgliche Leiden“ möglichst sicher feststellen, um lege artis - die Indikation für eine palliative Sedierung zu stellen oder gar

- einer Lebensbeendigung auf Verlangen oder - einem ärztlich assistierten Suizid zuzustimmen, zumal er auch rechtlich seine Indikation und Beurteilung verantwortet.

Unsichere Beurteilung I Natürlich sind Symptome und Situationen denkbar, die die meisten Menschen als leidvoll oder unerträglich bewerten würden. Es gibt aber auch eine große Grauzone, in der diese intersubjektive Evidenz fehlt. Wohl kann man sich bis zu einem gewissen Grad in die Lage eines anderen versetzen und dessen Leiden indirekt nachvollziehen [18]. Eine sichere Basis ist dies aber nicht, wie empirische Studien aus den Niederlanden zeigen.

- So verstanden Patienten, Angehörige und medizinische Fachkräfte unter einem „unerträglichen Leiden“ oft etwas Unterschiedliches. Ärzte bezogen sich meistens auf physische Symptome - während die Patienten sagten, eher unter psycho-sozialen und existenziellen Leidenserfahrungen gelitten zu haben $[11,29]$.

- In einer weiteren Studie gab mehr als die Hälfte der Ärzte an, unsicher zu sein bei der Beurteilung, ob ein unerträglicher Leidenszustand bei Sterbewilligen vorliege. Mehr als ein Viertel gab an, nicht bestimmen zu können, ob der Patient selbst das Leiden als unerträglich empfand [4].

Es bestehen erhebliche Schwierigkeiten beim objektiven, sicheren Assessment des individuellen Leidens. Dies führt zu großen Unsicherheiten. Besondere Schwierigkeiten bestehen bei Patienten, die ihr Leiden (z. B. aufgrund einer Demenz) nicht eindeutig artikulieren können [15].

Existenzielle Leidenserlebnisse | Aus dem subjektiven Charakter des Leidens folgt die nächste Frage: Welche Symptome und Situationen werden mit dem Begriff des Leidens erfasst? Geht es um körperliche Symptome wie Schmerz, Übelkeit oder Fatigue? Sind psychische Phänomene wie z.B. Depressivität gemeint? Oder auch existenzielle Erfahrungen wie Verzweiflung, Lebensüberdruss oder Trauer? Geht es um soziale Leiderfahrungen wie Einsamkeit oder die Angst, anderen zur Last zu fallen? Die niederländische Gesetzgebung hat diese Frage zunächst offen gelassen.

Rechtliche Entscheidungen | Einzelne Gerichtsurteile haben den Begriffsrahmen im Nachhinein etwas geschärft. Seit dem Fall Chabot ist klar: 
- Nicht nur physisches, sondern auch psychisches Leiden muss berücksichtigt werden, und

- ein Patient muss sich nicht in der Endphase einer terminalen Erkrankung befinden, um Hilfe zum Sterben zu erhalten.

- Das Urteil im Fall Brongersma definierte das existenzielle Leiden am „Lebensüberdruss“ vorerst als nicht berücksichtigungswürdig und forderte somit eine physische oder psychische Grunderkrankung als Ursache des Leidens [31].

Die Praxis zeigt: Auch soziale, existenzielle und spirituelle Zustände, die als unerträglich leidvoll geschildert werden, werden zunehmend als legitimierend für die Sterbehilfe im Einzelfall akzeptiert.

Gerade ältere aber nicht kranke Menschen bitten zunehmend Ärzte um Sterbehilfe [19]. Die Sterbeorganisation „Exit Deutsche Schweiz“ hat im Frühjahr 2014 begonnen, ihre Satzung zu ändern. Ziel ist, auch älteren Menschen, die nicht krank sind, Hilfe beim Suizid leisten zu können [13].

Erweiterung der Indikation | Auch in der Palliativmedizin gibt es seit einigen Jahren den Trend, die Indikation auf psychosoziale und existenzielle Leidenserlebnisse auszuweiten. Als psycho-existenzielle Leidenserlebnisse nennt die Literatur u. a. ein Gefühl von Sinnlosigkeit, die Angst, anderen zur Last zu fallen, Abhängigkeit, Angst vor dem Tod, Kontrollverlust, Einsamkeit oder ein Gefühl von Isolation $[28,20,26,9]$.

- Der breit gefächerte Leidensbegriff und seine Erweiterung auf psycho-existenzielle, soziale und spirituelle Leidenserlebnisse zeigen noch deutlicher, wie schwierig die intersubjektive Vermittelbarkeit des subjektiven Leidens ist.

- Symptome wie Schmerzen, Fatigue oder Dyspnoe lassen sich messen - offen ist, wie ein psycho-existenzielles, soziales oder spirituelles Leiden erfasst werden kann [28].

Zu seelischen und existenziellen Empfindungen haben Außenstehende nur sehr bedingt Zugang. Dies gilt umso mehr, wenn ökonomische Zwänge immer weniger Zeit für Zuwendung zum Patienten lassen.

Empirische Studien aus den Niederlanden zeigten, dass Ärzte das physische Leiden relativ ähnlich beurteilten - in der Beurteilung psycho-existenzieller Leiderfahrung gab es große Unterschiede $[29,30]$.

Internationale Uneinigkeit I Im internationalen palliativmedizinischen Diskurs sowie in den einschlägigen Leitlinien und Empfehlungen besteht Unsicherheit über das genaue Verständnis des Leidensbegriffs. Sollen psycho-existenzielle Leid- erfahrungen als Indikation für eine tiefe und kontinuierliche Sedierung gelten - oder nicht?

- Die American Medical Association (AMA) etwa sieht die palliative Sedierung nur zur Linderung refraktärer körperlicher Schmerzen und klinischer Symptome (Erbrechen, Atemnot, Delir) vor [34].

- Die Europäische Vereinigung für Palliative Care (EAPC) sieht hingegen vor, die palliative Sedierung auch zur Behandlung psychologischen und existenziellen Leidens am Lebensende anzuwenden [8].

Begriff der „Unerträglichkeit“ | Nicht nur der Leidensbegriff, auch das Adjektiv „unerträglich“ ist unzureichend bestimmt - was ähnliche Probleme aufwirft. Offensichtlich kann auch hier nur der Leidende die „Unerträglichkeit“ seines Zustands subjektiv definieren. Eine objektive Beurteilung von außen ist nicht möglich.

- Das Adjektiv „unerträglich“ verstärkt den appellativen und normativen Charakter des Leidensbegriffs. Es betont die Dringlichkeit einer Intervention, wodurch Handlungsdruck entsteht.

Die starke Normativität des „unerträglichen Leidens“ wirft Probleme auf - etwa das Risiko, dass das Leiden als moralisches Druckmittel zur Durchsetzung eigener Forderungen eingesetzt wird.

Zugespitzt könnte man fragen: Darf ein Arzt überhaupt das subjektive „unerträgliche Leiden“ eines Patienten hinterfragen? Strenggenommen müsste eine „Unerträglichkeit“ - aufgrund der darin ausgedrückten Dringlichkeit - alle Überprüfungsversuche schon aus Zeitgründen ausschließen.

Patientenautonomie I Es stellt sich zudem die Frage, wie Selbstbestimmung, Autonomie und unerträgliches Leiden zusammenhängen. Das wichtigste Kriterium der niederländischen Gesetzgebung ist, dass ein Patient seine Bitte um Sterbehilfe „freiwillig und nach reiflicher Überlegung“ stellen muss. Auch für eine als ultima ratio definierte Maßnahme - wie die terminale Sedierung - ist die Zustimmung des Patienten nötig. Doch ist ein Mensch, der „unerträglich leidet“, zu einem solchen Reflexionsprozess imstande? Schränkt ein unerträgliches Leiden die Fähigkeit zur Selbstbestimmung ein?

Erstes oder einziges Kriterium? | Die „Unerträglichkeit“ des Leidens kann dazu führen, dass andere Sorgfaltskriterien obsolet werden. Weshalb sollte eine Maßnahme zur Linderung von unerträglichem Leiden überhaupt noch an andere Sorgfaltskriterien (wie etwa das Vorliegen einer terminalen Erkrankung) gebunden sein? Die meisten Leitlinien zur palliativen Sedierung und die meisten Gesetze sowie aktuell hierzulande diskutierten Gesetzesvorschläge zur Regelung des ärztlich assistierten Suizids sehen aber diese 


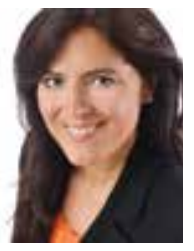

Dr. Claudia Bozzaro Mitarbeiterin am Institut für Ethik und Geschichte der Medizin, Universität Freiburg bozzaro@egm.uni-freiburg.de arbeitet als Wissenschaftliche

Sorgfaltskriterien vor. Ein Beispiel: Ein Patient im frühen Stadium einer terminalen Erkrankung bewertet seinen Zustand - zwar nicht krankheitsbedingt, aber dennoch - als „unerträglich leidvoll“. Warum sollte man ihm eine Sedierung verwehren, wenn die Perspektive besteht, dass dieses Leiden noch lange anhalten wird?

Tatsächlich zeichnet sich in Ländern mit etablierter Palliativ-Versorgung ein Trend zur sogenannten „early terminal sedation“ [7] ab. Diese früh praktizierte Form der tiefen kontinuierlichen Sedierung ist nicht unmittelbar an das absehbare Eintreten des Sterbeprozesses gebunden.

Die „early terminal sedation“ ist umstritten. Der Patient kann im frühen Erkrankungsstadium in die Sedierung einwilligen und zugleich auf lebenserhaltende Maßnahmen (Nahrung- und Flüssigkeitszufuhr) verzichten. Die Sedierung wäre dann eine „verdeckte oder verlangsamte Form“ („slow euthanasia“) aktiver Sterbehilfe $[2,12]$ oder ein „ärztlich assistierter Tod“ („physician assisted death“) [23]. Wie häufig die „frühe terminale Sedierung” derzeit vorkommt, ist nicht eindeutig dokumentiert. Kritiker meinen jedoch, dass auch ihr Einsatz zunehmen wird [7]

Auslegungssache | Die niederländische Gesetzgebung spricht nicht von terminaler Krankheitsoder Sterbephase, sondern von einer „aussichtslosen Situation“. Wieder fehlt eine Definition. Man könnte zynisch einwenden, dass jeder Mensch in einer aussichtslosen Situation ist, zumal wir von Geburt an dem Tode geweiht sind. Wenn man nun darunter „unerträglich leiden“ und davon einen Arzt überzeugen würde, so wäre theoretisch die Bedingung für Tötung auf Verlangen erfüllt.

- Interessant ist hier die jüngste Entscheidung des Brüsseler Berufungsgerichts. In Belgien ist seit 2002 - unter bestimmten Kriterien - die Tötung auf Verlangen straffrei. Das Gericht gestattete einem Sexualstraftäter die Sterbehilfe. Der Mann hatte sich als weiterhin gefährlich eingestuft und bezeichnete nach 30 Haftjahren sein Leben als „unerträgliche psychische Qual“.

Der Fall treibt das Beurteilungsproblem bei „unerträglichem/aussichtslosem Leiden“ auf die Spitze: Ist ein lebenslang Inhaftierter in einer ähnlich ausweglosen Situation wie ein terminal Erkrankter? War das Leiden des Häftlings ein Leiden, das mit ärztlicher Hilfe behoben werden sollte?

Die Autorin erklärt, dass keine Interessenkonflikte bestehen.

DOI 10.1055/s-0041-100030 Dtsch Med Wochenschr 2015; 140: 131-134

(c) Georg Thieme Verlag KG .

Stuttgart - New York .

ISSN 0012-0472
Fazit | Der Begriff des „unerträglichen Leidens“ in der medizinischen Praxis ist aus mindestens 3 Gründen problematisch:

1. Leiden ist ein absolut subjektives Erlebnis, das nicht mit Kriterien der Objektivität und intersubjektiver Vermittelbarkeit vereinbar ist.

2. Der Begriff ist inhaltlich nicht ausreichend differenziert - die Grenzen des medizinischen Linderungsauftrags sind unklar.

3. Der Leidensbegriff, insbesondere der des „unerträglichen Leidens“, ist normativ stark aufgeladen. Es besteht die Gefahr, dass andere Sorgfaltskriterien an Relevanz verlieren und der Begriff als moralisches Druckmittel missbraucht wird.

\section{Schlussbemerkung: Ärztlich assistierter Suizid bei „unerträglichem Leiden“?}

Öffentlicher Diskurs | Die genannten Beispiele aus Palliativmedizin und niederländischer Gesetzgebung haben den Klärungsbedarf gezeigt.

- Für eine Klärung braucht es nicht nur die Erhebung empirischer Daten,

- sondern auch eine interne Verständigung über die Möglichkeiten des ärztlichen Leidenslinderungsauftrags.

- Zudem ist eine öffentliche Diskussion über den Umgang mit leidenden Menschen in Medizin und Gesellschaft nötig.

Auch in unserer individualistischen Gesellschaft leben die meisten Menschen in Beziehungen - und sind damit Teil der Gesellschaft. Wie die Gesellschaft über Leiden denkt und wie Sie mit Leidenden umgeht, beeinflusst daher das Erleben und die Interpretation des individuellen Leidens. Es ist daher eine gesamtgesellschaftliche Aufgabe zu diskutieren und zu definieren, wo die Grenzen des Leidenslinderungsauftrags der Medizin gesetzt werden sollten.

Zulassung des ärztlich assistierten Suizids? I Wenn die ärztliche Beihilfe zum Suizid für die Linderung von „unerträglichem Leiden“ gesetzlich legitimiert wird, muss man aufgrund der beschriebenen Charakteristika des Begriffes des „unerträglichen Leidens“ folgendes erwarten:

- Es wird immer Einzelfälle geben, die aus dem Raster herausfallen und trotzdem ihren Anspruch auf Leidenslinderung geltend machen.

- Alle anderen Sorgfaltskriterien werden dadurch immer wieder infrage gestellt.

Dass eine gesetzliche Regelung somit Klarheit und Transparenz für die medizinische Praxis bringt, scheint mir äußerst unwahrscheinlich. Wahrscheinlicher ist längerfristig das Risiko einer Instrumentalisierung der Medizin und der Ärzte und dies für Zwecke, die mit der ärztlichen Kunst der Fürsorge und Hilfeleistung nur noch wenig zu tun haben. 


\section{Literatur}

1 Beijk M. Unbearable suffering: What Is It, what causes it, and who determines It? Medisch Contact 1998; 53: 825-827

2 Billings JA, Block SD. Slow euthanasia. J Palliative Care 1996; 12: 21-30

3 Bundesärztekammer. Grundsätze der Bundesärztekammer zur Sterbebegleitung. Dtsch Ärztebl 2011; 108: A346-A348

4 Buiting $\mathrm{H}$, Gevers ], Rietjens ] et al. Dutch criteria of due care for physician-assisted dying in medical practice: a physician perspective. J Med Ethics 2008; 34: e12

5 Callahan D. The goals of medicine: Setting new priorities. Hastings Center Report 1996; 26: Special Supplement

6 Cassel EJ. The nature of suffering and the goals of medicine. Oxford 1991

7 Cellarius V. „Early terminal sedation“ is a distinct entity. Bioethics 2011; 25: 46-54

8 Cherny NI, Radbruch L, The Board of EAPC. European Association for Palliative Care (EAPC) recommended framework for the use of sedation in palliative care. Palliative Med 2009; 23: 581-593

9 Claessens P, Menten J, Schotsmans P. Palliative sedation: A review of the research literature. J Pain Symptom Management 2008; 36: 310-333

10 DeGrazia D. Suffering: In Edward Craig (Hrsg.): Concise Routledge encyclopedia of philosophiy: Routledge 2000; 870

11 Dees M, Vernooij-Dassen M, Dekkers W et al. Unbearable suffering of patients with a request for euthanasia or physician-assisted suicide: An integrative review. Psycho-Oncology 2010; 19: 339-352

12 Delden van J. Terminal sedation: Source of a restless ethical debate. J Med Ethics 2007; 33: 187-188

13 EXIT Deutsche Schweiz: www.exit.ch/startseite. Letzter Zugriff 18.12.2014

14 Deutsches Referenzzentrum für Ethik in den Biowissenschaften. www.drze.de/im-blickpunkt/sterbehilfe/ rechtliche-regelungen. Letzter Zugriff 18.12.2014

15 Hertogh C. Unbearable suffering and advanced dementia: The moral problems of advance directives for euthanasia. In: Youngner S, Kimsma G (Hrsg.): Physician-Assisted Death in Perspective. Assessing the Dutch Experience. Cambrige, 2014; 215-228

16 Jones DA. Death by equivocation: a manifold definition of terminal sedation. In: Sterckx S, Raus K, Mortier F (Hrsg.): Continuous sedation at the end of life. Ethical, clinical and legal perspectives. Cambridge, 2013; 47-64

17 Juth N, Lindblad A, Lynöe $\mathrm{N}$ et al. European Association for Palliative Care (EAPC) framework for palliative sedation: An ethical discussion. BMC Palliative Care, 2010; 9: 20 doi:10.1186/1472-684X-9-20

18 Kimsma G. Assessment of unbearable andhopeless suffering in evaluating a request to end life. In: Youngner S, Kimsma G (Hrsg.): Physician-Assisted Death in Perspective. Assessing the Dutch Experience. Cambridge, 2014 ; 333-350
19 Lindemann, M. Zur Rechtswirklichkeit von Euthanasie und ärztlich assistiertem Suizid in den Niederlanden. In: Zeitschrift für die gesamte Strafrechtswissenschaft 2005; 117: 208-235

20 Morita T. Palliative sedation to relieve psycho-existential suffering of terminally ill cancer patients. J Pain Symptom Management 2004; 28: 445-450

21 Müller-Busch HC. Sterbende sedieren? Dtsch Med Wochenschr 2004; 129: 701-704

22 Müller-Busch HC, Radbruch L, Strasser F et al. Empfehlungen zur palliativen Sedierung. Dtsch Med Wochenschr 2006; 131: 2733-2736

23 Rady M, Verheijde J. Continuous deep sedation until death: Palliation or physician-assisted death? Amer J Hospice Palliative Med 2010; 27: 205-214

24 Sauer K. Palliative Sedierung. Der Urologe 2007; 46: 26-29

25 Schildmann J, Schildmann E. Clinical and ethical challenges of palliative sedation therapy. The need for clear guidance and professional competencies. Int ] Clin Pract 2013; 67: 1086-1088

26 Schuman-Olivier Z, Brendel D, Forstein M. The use of palliative sedation for existential distress: A psychiatric perspective. Harvard Rev Psychiat 2008; 16: 339-351

27 Sterckx S, Raus K, Mortier F. Introduction. In: Sterckx S, Raus K, Mortier F (Hrsg.): Continuous sedation at the end of life. Ethical, clinical and legal perspectives. Cambridge, 2013; 1-28

28 Swart S], van der Heide A, van Zuylen L et al. Continuous palliative sedation: Not only a response to physical suffering. J Palliative Med 2014; 17; 27-36

29 Tol van D, Rietjens J, van der Heide A. Judgment of unbearable suffering and willingness to grant a euthanasia request by Dutch general practitioners. Health Policy 2010; 97: 166-172

30 Tol van D, Rietjens J, van der Heide A. Empathy and the application of the ,unbearable suffering criterion in Dutch euthanasia practice. Health Policy 2012; 105: 296-302

31 Weyers, $\mathrm{H}$. The Legalization of Euthanasia in the Netherlands: Revolutionary Normality. In: Youngner S, Kimsma G (Hrsg.): Physician-Assisted Death in Perspective. Assessing the Dutch Experience. Cambridge, 2014; 34-68

32 Wijsbek $H$. The Subjectivity of Suffering and the Normativity of Unbearableness. In: Youngner S, Kimsma G (Hrsg.): Physician-Assisted Death in Perspective. Assessing the Dutch Experience. Cambridge, 2014; 319-332

33 Bozzaro C. Der Leidensbegriff im medizinischen Kontext. Ein Problemaufriss am Beispiel der tiefen palliativen Sedierung am Lebensende. In: Ethik in der Medizin (im Erscheinen)

34 CEJA Report der American Medical Association (2008) Sedation to Unconsciousness in End-of-Life Care. http://www.ama-assn.org/resources/doc/ethics/x-pub/ ceja_5a08.pdf. Letzter Zugriff 4.10.2014 